\title{
Percolation-induced conductor-insulator transition in a system of metal spheres in a dielectric fluid
}

\author{
Gang Sun and Kunquan Lu \\ Beijing National Laboratory for Condensed Matter Physics and Key Laboratory of Soft Matter Physics, \\ Institute of Physics, Chinese Academy of Sciences, Beijing 100190, China \\ Ferenc Kun \\ Department of Theoretical Physics, University of Debrecen, P. O. Box 5, H-4010 Debrecen, Hungary
}

(Received 2 July 2010; revised manuscript received 6 December 2010; published 15 April 2011)

\begin{abstract}
We develop a model to investigate the insulator-conductor transition observed in a system of spherical metal particles suspended in a quasi-two-dimensional viscous liquid between planar electrodes when the voltage of the electrodes is increased. Our model captures the main ingredients of the process in experimental system, and reveals the insulator-conductor transition at a well-defined critical voltage. Based on the simulation data we demonstrate that characteristic quantities of the system show power-law scaling in the vicinity of the critical point. These scaling analysis show clearly that the transition between the insulating and conducting phases is analogous to second-order phase transitions.
\end{abstract}

DOI: 10.1103/PhysRevE.83.041405

PACS number(s): 82.70.Dd, 83.80.Gv, 64.60.ah

\section{INTRODUCTION}

Phase transition and critical phenomenon is a field facing many difficulties and challenges [1-3]. Phase transitions can result in from not only physical changes but also geometrical changes. The most known examples are the Ising ferromagnet [4-6] and the random resistor network [7-9]. For these systems, it has been proposed [10-14] that the scaling behavior of the effective conductivity of the network of resistors is analogous to that exhibited by the magnetization of an Ising ferromagnet. The concentration of the lower resistors plays the role of temperature and its percolation threshold corresponds to the Curie temperature. The applied magnetic field in an Ising ferromagnet is replaced by the ratio of the conductivity of higher resistors to that of the lower ones. The exact mapping of the two systems lets the random resistor network be well understood, and by now it is known as a fundamental model for geometrical phase transitions [2].

The random resistor network is an ideal theoretical model, in which the distribution of resistors is always random. However, in the real world, a broad class of phenomena involves disordered connectedness whose structure varies due to external driving. For example, dielectric particles in electrorheological (ER) fluids aggregate and form chainlike clusters when an electric field is applied on the system, which then results in a prominent increment of the yield stress [15-19]. However, it is difficult to show that ER fluids have a macroscopic phase transition, although the formation of the chainlike structure shows some scaling behavior [20,21]. In general, ER fluids are composed of dielectric dispersed particles and dielectric dispersing liquid, i.e., both components are nonconducting materials, so the ratio of the conductivity of higher resistors to that of the lower ones is not controllable. This violates the condition that the ratio tends to zero when a macroscopic insulator-conductor transition occurs. However, if one replaces the dielectric dispersed particles with metal particles, the characteristic of chain formation in ER fluids will be retained but its effective conductivity will show an insulator-conductor phase transition [22], similar with that of the random resistor network. Therefore, the metal-oil ER fluid system is a good candidate to study the phase transition dominated by the disordered connectedness which varies in response to external control.

In this paper we present a model of metal-oil ER fluids to investigate the two main characteristics observed in experiments on ER systems, i.e., the gradual formation of chainlike clusters and the insulator-conductor transition with increasing external voltage. Experiments have revealed that, similar to dielectric ER fluids, metal-oil ER fluids also change their symmetry very much when an electric field is applied [22]. As a representative example, the snapshot of the evolution of the microstructure of the metal-oil ER system is presented in Fig. 1(a), which is cited from Fig. 2(a) in Ref. [22]. In this figure, the volume fraction is 0.082 . Under the assumption that the fluid is just as thin as the diameter of the uniform metal spheres, the two-dimensional (2D) volume (section area) fraction is $3 / 2$ times this value, i.e., 0.123 . However, the metal spheres are not uniform in the experiment and it is also not possible to control the thickness of the oil just at the diameter of the metal spheres. For this reason, the 2D volume fraction should be somewhat higher than this value. Figure 1(a) shows clearly that the metal spheres form a chainlike structure with many branches when strong electric field is applied. To observe a fractal structure with many branches, large system sizes are absolutely needed. In order to make large-scale computer simulations feasible, some simplifications had to be carried out in the model construction: The main simplifications we used are the discretization of the position of metal particles and we replaced the inhomogeneous media by a resistor network. These simplifications allow us to calculate both the distribution of the field and the whole current of the system very efficiently by solving Kirchhoff's equation. Benefiting from the efficient algorithm, we can treat systems with particle number much larger than in the experiments in Ref. [22]. The paper is organized as follows: In Sec. II we briefly describe the model used and the simulation method. Section III presents the simulation results, which show clearly the emergence of an insulator-conductor transition as the 

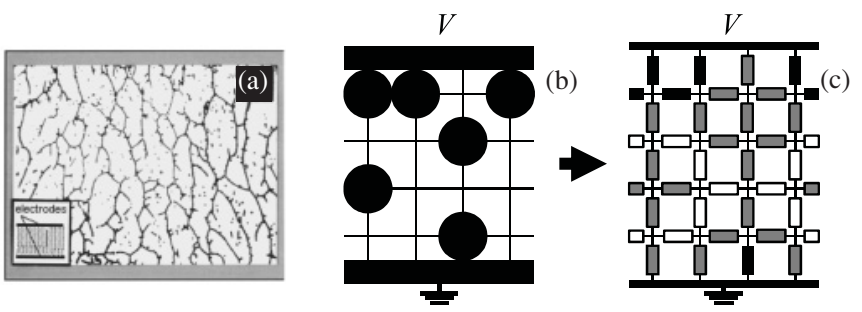

FIG. 1. Snapshots of the experimental system at high voltage values obtained in the experiments of Ref. [22]. The snapshot (a) shows clearly that the particles form chains which merge into a large cluster spanning between the electrodes. The model construction is illustrated by (b) and (c).

electric voltage is increased. In Sec. IV the cluster structure is analyzed in detail and the connection of the microstructure to the insulator-conductor transition is established. Section V is devoted to a conclusion and discussion.

\section{SIMULATION METHOD}

The goal of our work is to carry out a detailed investigation of the insulator-conductor phase transition occurring in metal-oil electrorheological fluids, focusing also on the microstructural change of the system when the external voltage is increased. In the system, metal particles are dispersed in a fluid between two planar electrodes which makes it possible to apply a controllable external electric field. The basic mechanisms occurring in this physical system can be summarized as follows: When the electric potential is applied on the two electrodes on opposite ends of the system, an inhomogeneous electric field will form. The electric field will further induce a dipole moment $\vec{d}=\alpha \vec{E}$ for each isolated metal sphere (not directly or indirectly connected to the electrodes), where $\alpha$ is a material-dependent parameter and $\vec{E}$ denotes the field strength at the location of the particle. The inhomogeneous electric field exerts a force on the metal spheres with induced dipole moment, which can be written in the form

$$
\vec{F}=(\vec{d} \cdot \vec{\nabla}) \vec{E}
$$

This electric force will drive the isolated metal spheres to move. As a consequence the particles aggregate and form a chainlike pattern in the fluid as the electric voltage increases. Under this consideration, two arduous works have to be done in the simulation, i.e., the inhomogeneous electric field has to be calculated, taking also into account the effect of the induced dipole moments, and the resulting motion of the metal spheres has to be determined in the fluid.

It has been mentioned that many-body effects are dominant in this system, so a very large number of metal spheres has to be treated in order to obtain reliable results. In recent years, there are significant developments in the simulation techniques for the soft-matter systems [23-25]. However, for the calculations on the electrostatics of the systems composed of particles, the most efficient methods are based upon the particle-particle-particle-mesh algorithm [26,27], which is not enough in treating the system with the size we used in this paper. To obtain the electrostatics, we generally solve Maxwell equations with a complex permittivity, which combines the information of permittivity and conductance together. If the system contains only dielectric materials, a real permittivity is enough to describe the system, and Maxwell equations reduce to the Poisson equation. However, if the system contains metal materials, the free charge will be dominated, and the field distribution is determined by the irrotational properties of the electric field, which results in Kirchhoff's equation. To simplify the system so that it is suitable for this kind of calculation, we discretize the system and restrict the position of the metal spheres on the nodes of a square lattice [Fig. 1(b)]. Then, the distribution of the electric media is approximated by a network of resistors on the square lattice, which is formed by connecting each nearest-neighbor site with a resistor. Figure 1(c) is an illustration of the approximation for the configuration shown in Fig. 1(b). There are three kinds of resistors in the network corresponding to three different kinds of configuration in the nearest-neighbor sites, i.e., $R_{m}$ [black resistor in Fig. 1(c)] for both of the sites being occupied by metal spheres, $R_{i}$ [white resistor in Fig. 1(c)] for both being empty, and $R_{m i}=R_{m} / 2+R_{i} / 2$ [gray resistor in Fig. 1(c)] for one site being occupied and the other being empty. Here, it is should be pointed out that large but finite $R_{i}$ is needed to ensure that the whole system has a stable solution. After this simplification, the distribution of the electric potential when a fixed voltage is applied at the two opposite edges of the square lattice can be calculated by solving Kirchhoff's equation for each node [28]. The inhomogeneous electric field can be obtained from the electric potential as

$$
\vec{E}=-\vec{\nabla} V
$$

The other component of the simulation is to determine the rule of movement of the metal spheres. After the inhomogeneous electric field is obtained, it is easy to calculate the electric force exerted on each metal sphere according to Eq. (1). However, in the process of chain formation, the fluid also exerts force on the metal spheres. The simulation rule for the movement of the metal spheres must include the effect of this hydrodynamic force. Some previous work has used Stokes' law to describe the hydrodynamic force [29]. However, because we discretize the positions of the spheres, an accurate velocity cannot be obtained. Fortunately, the movement of the metal spheres is slow, so the Stokes force can be neglected compare to another frictionlike force, which is caused by the contact of the metal spheres and the bottom plate of the container. The frictionlike force will keep the metal sphere in its original site until the component of the force on a specific direction exceeds a threshold value $f_{0}$. When a force component is larger than the threshold, the metal sphere will hop to its nearest-neighbor site along the direction if there is an empty (not occupied by another metal) sphere). Sometimes there may exist multiple directions that satisfy the above condition, and in this case the metal sphere will hop to the direction with the largest force component. Since the velocity of the sphere is neglected in our simulation, the hopping is restricted only to its nearest-neighbor site, regardless of the strength of the electric force. The metal spheres that are directly or through other metal spheres connected to the electrode will not experience an electric force because of the screening effect. Therefore, we also exclude the consideration of the 
movement of these spheres in the simulation, as was done by Kun in Ref. [29]. This means that if a metal sphere moves to connect to a cluster that is connected to the electrode or to the electrode directly, it will be fixed there in the rest of simulation time.

After we have determined the voltage distribution of the network, it is trivial to calculate the electric current at each resistor by use of Ohm's law, and the current of the whole system can be obtained easily by summing up all these currents along the section perpendicular to the direction of the electric potential applied. As general, the effective conductivity of the whole system is defined as the ratio of the current of the whole system to the applied voltage.

The computer simulation of the model proceeds as follows: We start at a random configuration of metal spheres without any external electric field. Those metal particles that are directly or through other metal spheres connected to the electrodes are marked and are ruled out of the consideration of movement during the simulation. Then the electric voltage is gradually increased in equal steps. At each voltage value, the movement of all movable particles is determined according to the rules mentioned above. Those particles which get connected to the electrodes (directly or through other metal spheres) during the time evolution get fixed to the electrode. The trial movement process is iterated 100 times at each voltage to reach an equilibrium state. The conductivity of the system at each voltage is calculated after the equilibration process. The simulation is stopped at a very high voltage, where the conductivity of the whole system reaches its saturation value, or there are no movable metal spheres in the whole system.

The conditions of the simulations are set in the following way. Our simulation is carried out on the $L \times(L+2)$ square lattice in the range $L=200 \rightarrow 1000$. A fixed boundary condition is used in the longer direction to simulate the effect of the electrodes, where two edges are fully filled by metal spheres, and its voltage is set to 0 and $V$, respectively. For the shorter direction, a periodic boundary condition is simply used. It is worth mentioning that the results of the conductivity may fluctuate for different random initial configurations, especially for a smaller system size. To ensure reasonable accuracy, we carried out the simulation five times with a different random initial position of the particles at each system size, and the final conductivity at each voltage was obtained by averaging the simulation results.

In our model construction the system is characterized by a large number of parameters. However, we can find that some of them merely give scale variations, but do not give a different physical description. We first consider the parameters $R_{m}$ and $R_{i}$. We can find that the distribution of the electric potential depends only on the ratio of the two kinds of resistance $R_{i} / R_{m}$. If we fix the ratio and change $R_{m}$ to $R_{m}^{\prime}$, the distribution of electric potential is exactly the same, and hence the simulation process is the same. The only difference is that the current on each resistor changes by a factor $R_{m} / R_{m}^{\prime}$, and hence the total current also changes by this factor. Second, we consider the rule of movement. In our movement rule, whether a sphere moves or not is determined by the comparison of the electric force and the frictionlike force. The change of the system is in a quasistatic process, because the effect of the velocity of the spheres is neglected. In this case, the precise value of the difference between the electric and frictionlike force is not important, i.e., it will give exactly the same movement if the logical comparison (less or larger) is unchanged. According to this, the key parameter should be $[(\vec{d} \cdot \vec{\nabla}) \vec{E}]_{i} / f_{0}$, which is proportional to $\alpha V^{2} / f_{0}$. This means that the same value of $\alpha V^{2} / f_{0}$ would show exactly the same movement of each sphere in the simulation. Furthermore, the conductivity-voltage relation will show similar characteristics (with different critical voltage) for any parameters of $\alpha$ and $f_{0}$ as long as they are positive, because the difference of $\alpha / f_{0}$ can be easily merged into the scale of the voltage.

According to the previous discussion, the only important parameter is the ratio of two kinds of resistance $R_{i} / R_{m}$, which is chosen to be $1.0 \times 10^{9}$ in our simulation. We choose this value by considering that there is a very thin fluid film between the two touching metal spheres, so the resistance between the two metal spheres in the fluid is much larger than the resistance in bulk metal. We have also carried out the simulations for other ratios of the resistance with fixed $R_{m}$. We find that the result of the conductivity-voltage relation is almost the same, provided the ratio is sufficiently large, e.g., $R_{i} / R_{m}>1.0 \times 10^{4}$. The value of $R_{m}$ is set to $1.0 \times 10^{-5} \Omega$ and the parameter $\alpha / f_{0}$ is set to $1.0 \times 10^{-2} \mathrm{~V}^{-2}$ for a unit length of the lattice or the sphere's diameter. The concentration of the metal spheres is chosen to be $\phi=30 \%$ per lattice site, which is $(\pi / 4) 30 \% \approx 23.6 \%$ for volume occupation.

\section{TRANSITION FROM THE INSULATING TO THE CONDUCTING PHASE}

Figure 2 presents the configuration of metal spheres obtained by computer simulations at different voltages. At zero voltage, $V=0$, the particles are located at random positions [Fig. 2(a)]. By increasing $V$, the attracting particles can hop to neighboring sites, which results in a cluster-cluster aggregation process with clusters elongated in the field direction [Figs. 2(b) and 2(c)]. At higher voltages the shape of the clusters becomes more anisotropic and at a critical voltage a spanning cluster appears which connects the two electrodes [Fig. 2(d)]. Even much above the transition points [Fig. 2(e)], there can be some isolated particles which have not joined the giant cluster. The evolution of the configurations as the voltage increases is similar to that of the experiment in Fig. 1(a) and also to that of the dielectric ER fluid experiments [30-32].

The conductivity-voltage relation is plotted in Fig. 3, where a transition can be observed from a high-resistance range at low voltages to a low-resistance range at high voltages. The transition from the insulator (high resistance) to the conductor state (low resistance) is relatively fast. This transition also depends on the concentration of the metal spheres. By slightly changing the concentration, we find that it needs a higher voltage to transform from the insulator to the conductor state for a system with a lower concentration. All these characteristic features of the system are in a good agreement with the experimental findings [22]. Our simulations show that the insulator-conductor transition emerges due to the change of the configuration of metal spheres in the space, as it is 

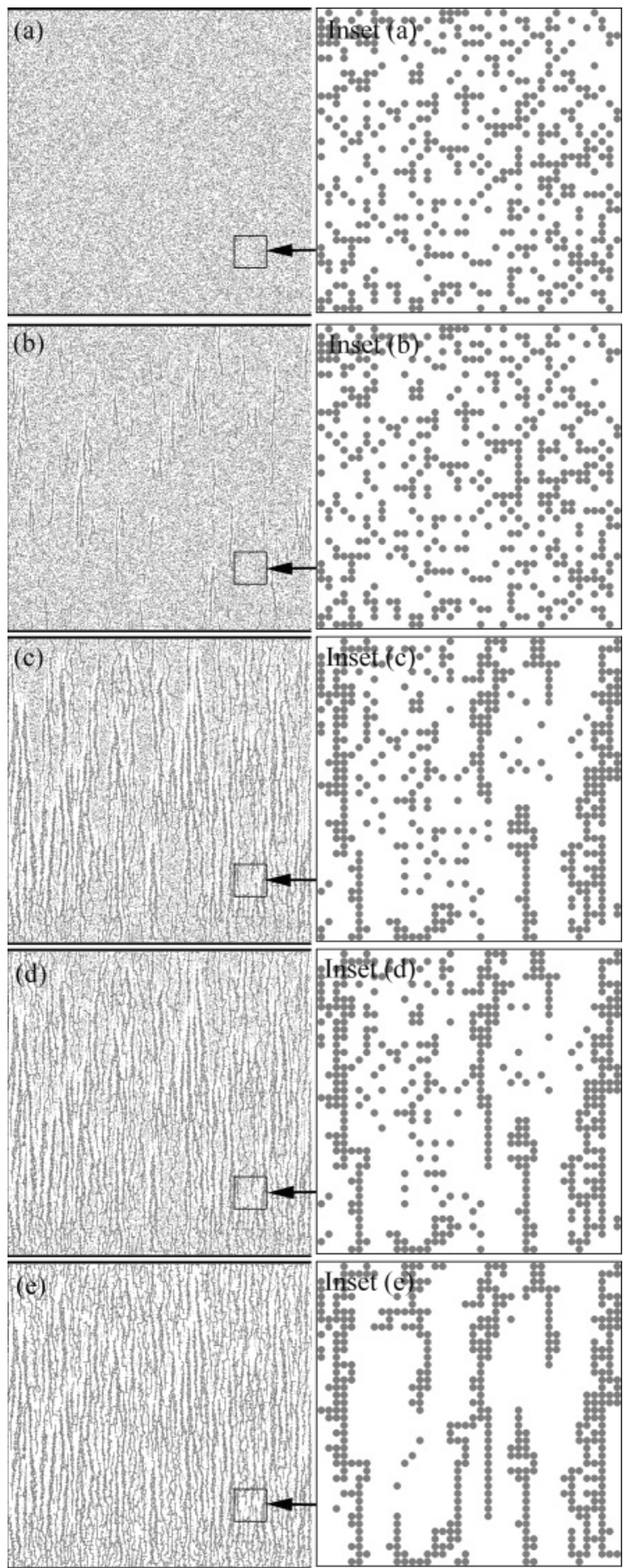

FIG. 2. Simulation configuration of the equilibrium state for the system of size $L=400$ at the voltage $V=$ (a) 0 , (b) 500 , (c) 600, (d) 700, and (e) 2400, respectively. The figures in the right-hand column present magnified views of the areas indicated by the rectangles.

demonstrated in Fig. 2. Comparing Figs. 2 and 3, it can be observed that the beginning of the transition corresponds to the formation of short chains of the metal spheres, and the fastest variation of the conductivity corresponds to the percolated structure, where the metal spheres just form a connected cluster between the two electrodes. The conductivity reaches

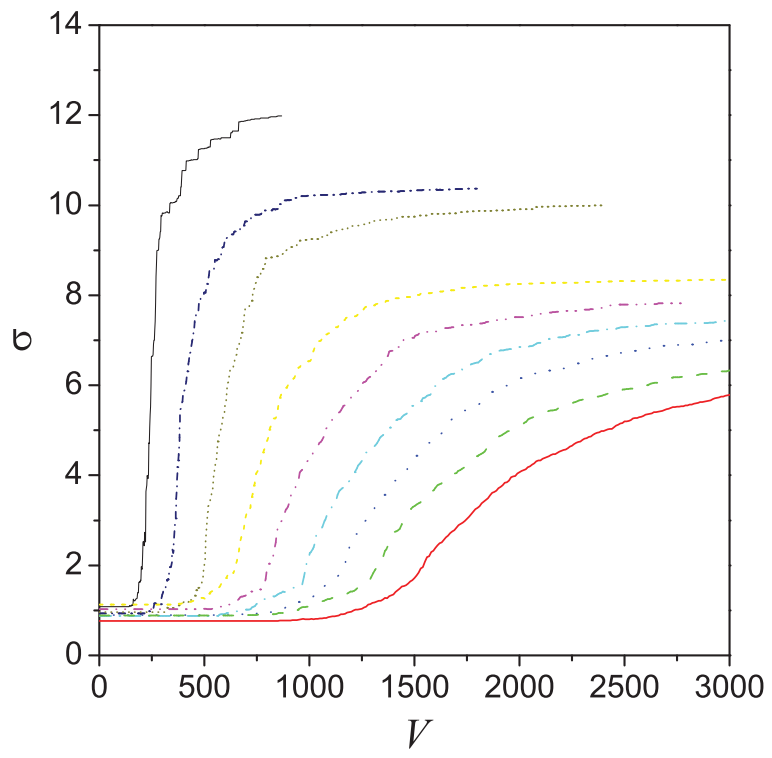

FIG. 3. (Color online) Conductivity $\sigma$ as a function of voltage $V$ for different system sizes $L$. The system size increases from up-left to the down-right as $L=200,300,400,500,600,700,800,900,1000$.

the saturation value when most of the metal particles aggregate to the chainlike structure spanning the entire space between the electrodes.

For such a percolation-induced insulator-conductor transition it is important to analyze how the system size affects the process. Figure 4 presents the saturation value of the conductivity $\sigma_{0}$ as a function of the system size on a double logarithmic plot. It can be seen that $\sigma_{0}$ is not a constant, as is generally found for 2D compact, space-filling systems, but decreases as the system size increases. This means that a larger fraction of the metal spheres touch the bifurcated part of the chainlike structure for larger systems, which will not contribute to the conductivity of the whole system. Hence the effective

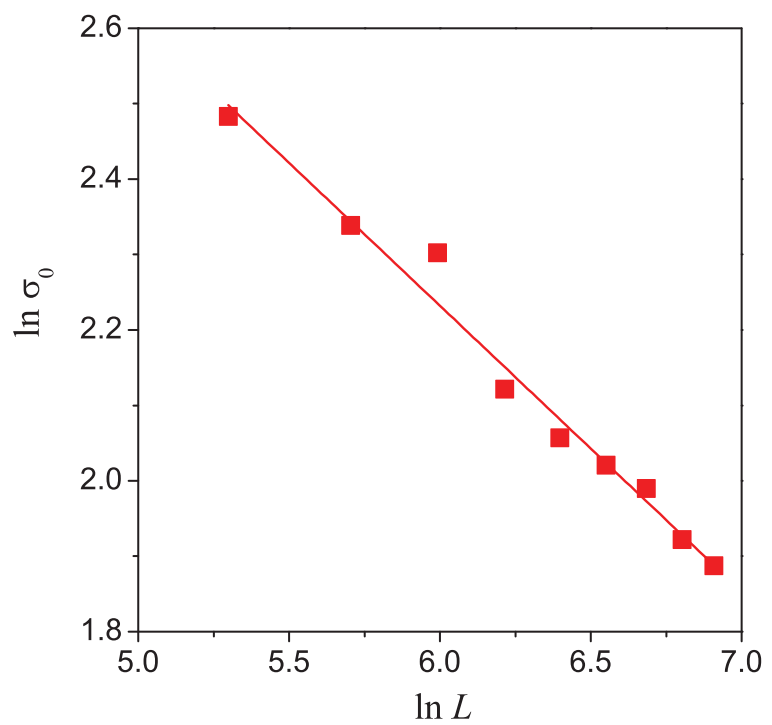

FIG. 4. (Color online) The saturation value of the conductivity $\sigma_{0}$ as a function of the system size $L$. Power law behavior is evidenced with a fractal section of the conducting backbone. 


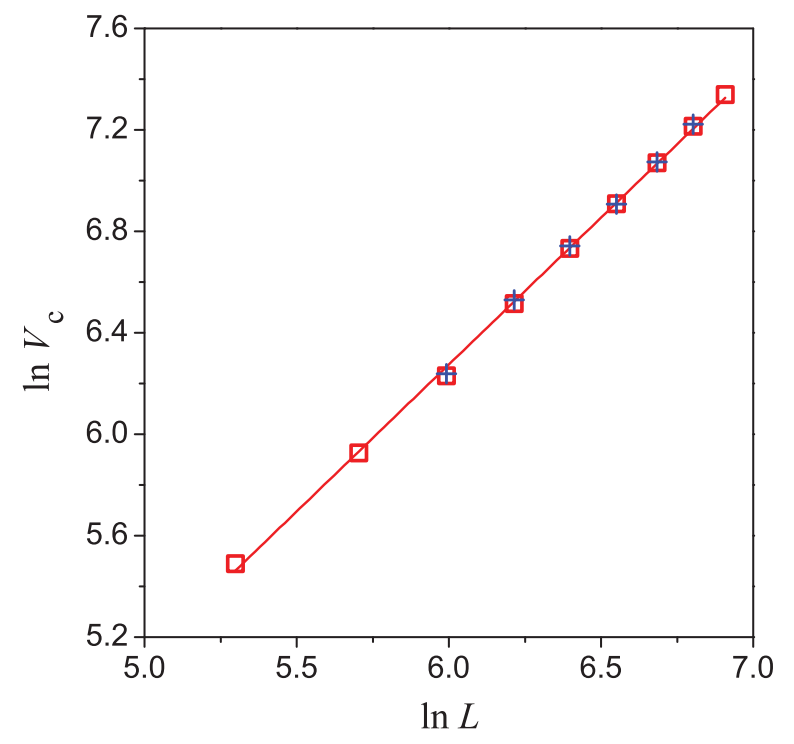

FIG. 5. (Color online) Critical voltage $V_{c}$ obtained by the fastest change of the conductivity (squares) and by the peak of the average cluster size (cross) as a function of the system size $L$. A high-quality power-law behavior is obtained with the exponent $1+\gamma$, where $\gamma=$ $0.23 \pm 0.02$ is for the data represented by squares.

number of the backbone per width decreases. The data in Fig. 4 can be well fitted by a straight line which implies the power-law behavior

$$
\sigma_{0} \sim L^{-\beta}
$$

where the value of the exponent was obtained as $\beta=0.38 \pm$ 0.07 , which suggests that the conducting backbone has an effective section $S_{\text {eff }}$ with a fractal dimension $1-\beta$.

The transition voltage $V_{c}$ of the system was determined as the voltage where the conductivity has the fastest change. Numerically $V_{c}$ was obtained by smoothing the conductivity curves of Fig. 3 and searching for the position of the maximum of the derivative. Figure 5 presents the $\ln -\ln$ plot of the transition voltage $V_{c}$ as a function of the system size $L$. It can be observed in the figure that the transition voltage increases with system size. Assuming that the transition voltage is determined by the electric-field strength $E, V_{c}$ should be proportional to the system size $L$. Figure 5 shows a power-law increase of $V_{c}$,

$$
V_{c} \sim L^{1+\gamma},
$$

where the exponent is proved to be $\gamma=0.23 \pm 0.02$. The nonzero value of $\gamma$ implies that the transition point is not simply determined by the strength $E$ of the electric field, since with increasing system size a larger fraction of the metal spheres are attached to side branches of the elongated clusters which do not contribute to the current-carrying capacity of the system. This is an interesting consequence of the fractal structure of clusters which couple the two electrodes.

\section{STRUCTURAL TRANSITION OF THE PARTICLE SYSTEM}

Studying macroscopic observables of the system, we have shown above that the system exhibits a transition from an insulating state to a conducting one appearing at a well-defined critical voltage. On the microlevel, the insulating-conducting transition is accompanied by subsequent rearrangements of the particles, which gives rise to a structural transition from an initially random configuration to an ordered one where a spanning cluster of metal spheres ensures conduction between the plates. In order to obtain a deeper understanding of the phase transition, in the following we present a detailed analysis of the structural evolution of the particle system as the voltage of the electrodes is increased.

In the initial configuration of the model system, the particles are placed randomly onto the nodes of the square lattice. Since the concentration $\phi$ is significantly below the corresponding site percolation threshold of the lattice, $\phi<\phi_{c} \approx 0.5927$ [33-35], the particles can only form clusters which are much smaller than the system size. The cluster size $S$ is defined as the number of particles in the cluster. As the voltage between the two electrodes increases, the particles and the small initial clusters start to move and form larger clusters. It can be observed in Fig. 2 that as the voltage increases, the system undergoes a cluster-cluster aggregation process: At low voltages only small clusters are present, which then grow by merging. Since the electrostatic interaction favors the clusters' ends for merging, the clusters get more and more elongated as they grow [see Figs. 2(b)-2(e)]. At the critical voltage $V_{c}$, this growth process gives rise to the emergence of a macroscopic cluster which spans the gap between the two electrodes, introducing a conducting contact between them. The conductivity of the system above the critical voltage $V>V_{c}$ in Fig. 2 is mainly determined by the structure of the conducting backbone of the spanning cluster of metal particles.

For the quantitative characterization of the structural evolution of the particle ensemble, we determined the radius of gyration $R_{g}$ of all growing clusters before they join one of the electrodes (directly or indirectly). The radius of gyration $R_{g}$ characterizing the linear extension of an $S$-sized cluster is defined as

$$
R_{g}^{2}=\frac{1}{S(S-1)} \sum_{i \neq j=1}^{S}\left(\vec{r}_{i}-\vec{r}_{j}\right)^{2},
$$

where $\vec{r}_{i}$ denotes the position vector of particle $i$ in the cluster. Figure 6 shows that the size of clusters $S$ has a power-law dependence on the radius of gyration $R_{g}$,

$$
S \sim R_{g}^{D},
$$

where the value of the exponent $D$ depends on the size of the clusters. Since small clusters are more compact, they are characterized by a high value of the exponent $D=$ $1.75 \pm 0.015$. Large clusters tend to merge at their ends, which gives rise to elongated cluster shapes with a lower value of the exponent $D=1.25 \pm 0.05$. It can be observed that the crossover between the two regimes occurs at a well-defined cluster size $S_{c} \approx 15$, indicated by the dashed line in the figure. It has to be emphasized that the value of $D$ significantly lower than 2 that was obtained for large clusters demonstrates the self-affine character of the shape of clusters growing in the inhomogeneous electric field. Assuming that the finite clusters in Fig. 2 can be enclosed by a rectangular bounding box of side length $a$ and $b$, the cluster size $S$ can be estimated as $S=a b$ and the radius of gyration can be obtained 


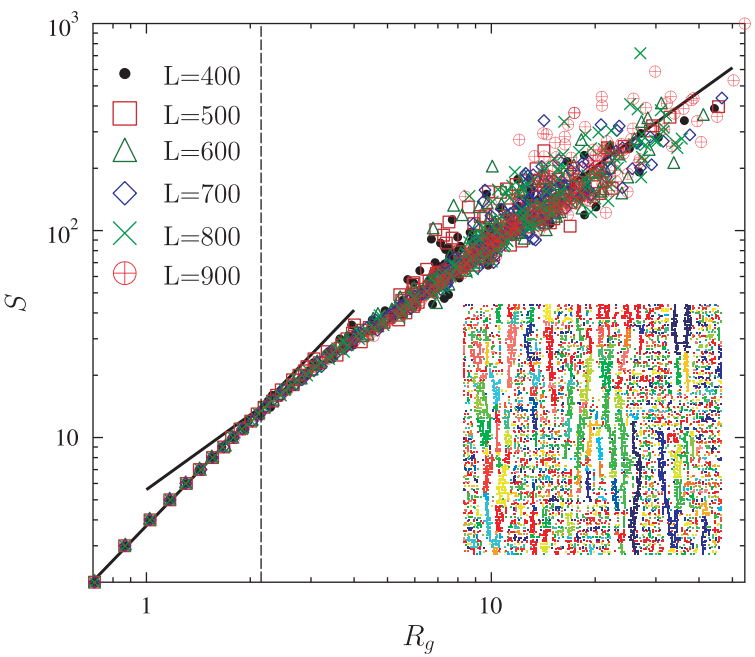

FIG. 6. (Color online) Size of the clusters $S$ as a function of the radius of gyration $R_{g}$ obtained at different system sizes $L$. Each symbol represents an individual cluster. On a double logarithmic plot, the data can be well approximated by straight lines. The inset presents a magnified view of the cluster structure.

as $R_{g}=\sqrt{a^{2}+b^{2}} /(2 \sqrt{3})$. The elongated shape of clusters results in a high value of the aspect ratio $\chi=a / b \gg 1$. Using $R_{g}$ and $\chi$ instead of the two side lengths $a$ and $b$, the cluster size can be expressed in the form $S=R_{g}^{2} /(\chi+1 / \chi)$, which can be well approximated by $S \approx R_{g}^{2} / \chi$. This argument has the consequence that the low value of $D$ can be explained such that, due to the strongly inhomogeneous electric field, the growing clusters become more and more anisotropic and the aspect ratio increases as a power law of $R_{g}$, i.e., $\chi \sim R_{g}^{\delta}$. The exponent $\delta$ is an important characteristic quantity of the ER system [20,21], it demonstrates that the clusters of the ER system have a self-affine character, i.e., the larger they get, the more anisotropic they are [36-38]. In our system $\delta=2-D$ gives rise to the value $\delta=0.75$ of the self-affinity exponent.

In order to understand the nature of the transition from the insulating to the conducting phase, we have to analyze how the ensemble of growing clusters evolves as the voltage is increased. We evaluated the size distribution of clusters $n_{V}(S)$ at different voltages, which provides the number of those clusters $n$ of different sizes $S$ that are not connected to the electrodes. It can be seen in Fig. 7 that at low voltages, when all clusters are small, the distribution has a rapid exponential decay. As the voltage approaches the critical point $V \rightarrow V_{c}$, due to the merging of growing clusters, the distribution spans a broader and broader range but still the exponential cutoff dominates the behavior of $n_{V}(S)$. It has to be emphasized that at the critical voltage $V_{c}$ the distribution becomes a power law,

$$
n_{V}(S) \sim S^{-\tau},
$$

where the value of the exponent $\tau=2.12 \pm 0.04$ was obtained numerically. In the supercritical regime $V>V_{c}$ the range of cluster sizes decreases again (see also Fig. 7) since an increasing fraction of particles joins the spanning cluster contributing to the conduction. Note that the value of $\tau$ falls relatively close to the cluster size exponent of $2 \mathrm{D}$ percolation

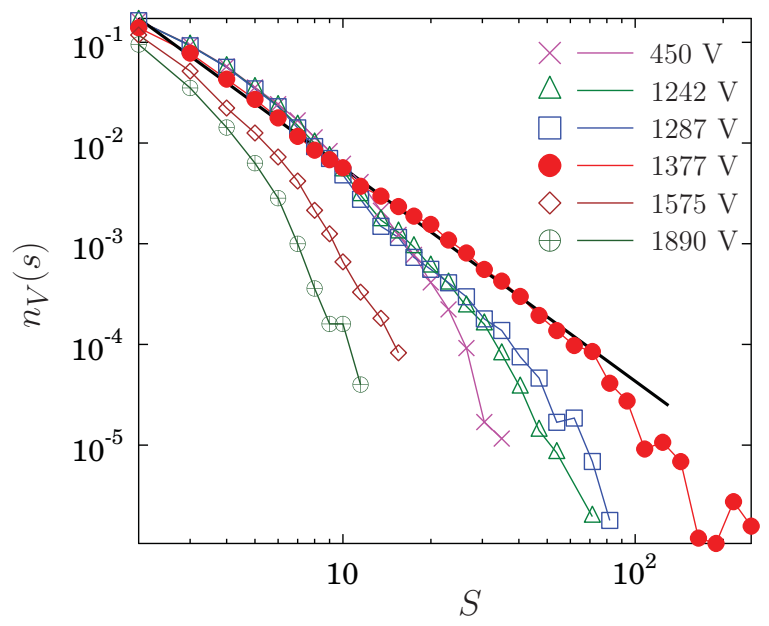

FIG. 7. (Color online) The size distribution of clusters $n_{V}(S)$ obtained at different voltages $V$ for the system size $L=900$. As the critical voltage is approached, $V_{c} \approx 1377 \mathrm{~V}$, the distribution becomes a power law. The slope of the straight line is 2.12 .

[9] in spite of the correlated growth and anisotropic shape of clusters.

The transition from the insulating to the conducting phase can be followed by studying the average size of finite clusters, i.e., the size of those clusters which are not connected to the electrodes. The average cluster size $S_{a v}$ is defined as the ratio of the second and first moments of the cluster size distribution,

$$
S_{\mathrm{av}}=\frac{\sum_{S}^{\prime} n_{V}(S) S^{2}}{\sum_{S}^{\prime} n_{V}(S) S}
$$

where the prime indicates that the largest cluster is always omitted in the summation [9]. The average cluster size is presented in Fig. 8 as a function of voltage for different system sizes $L$. It can be observed that, due to the coalescence of growing clusters, the average size of the clusters increases with the voltage and has a relatively sharp maximum, where the spanning cluster is formed. Since the large cluster becomes

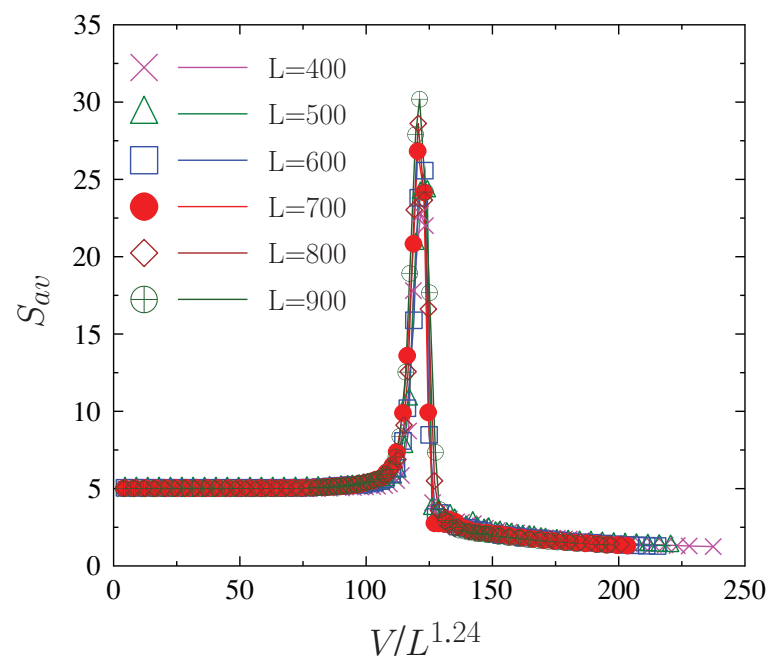

FIG. 8. (Color online) Average cluster size $S_{a v}$ as a function of voltage rescaled with the system size $L$. With an appropriate choice of the exponent of $L$, the curves can be collapsed onto each other. 
rapidly connected to the electrodes, the finite clusters become relatively small and $S_{\text {av }}$ decreases above the critical point $V>V_{c}$. Figure 8 also demonstrates that, by rescaling the voltage with an appropriate power of the system size, the $S_{\mathrm{av}}$ curves obtained at different system sizes can be collapsed on the top of each other. The good quality collapse of Fig. 8 was obtained with the exponent 1.24 , which is very close to the value of $1+\gamma$ obtained for the scaling of the transition voltage, Eq. (4). The scaling analysis implies that the position of the maximum $V_{m}$ of the average cluster size $S_{\text {av }}$ also has the system size dependence,

$$
V_{m} \sim L^{1+\gamma}
$$

Note that $V_{m}$ not only has a similar scaling behavior as $V_{c}$, but its value is also very close to $V_{c}$. For comparison, we also plot $V_{m}$ in Fig. 5. These cluster structure analysis results clearly support the argument that the conductor-insulator transition taking place in the system at the critical voltage has a percolation nature. The difference of the exponent $\tau$ and of the fractal dimension from their counterparts in 2D percolation on a square lattice [9] can be attributed to the spatial correlation arising due to the anisotropic growth of the clusters of metal particles. Due to large numerical costs, our scaling analysis is limited to the size scaling of the average cluster size $S_{\mathrm{av}}$ where good agreement is found with the scaling behavior of the conductivity.

\section{CONCLUSIONS}

We established a simple model to simulate the system that spherical metal particles are suspended in a quasi-2D viscous liquid between planar electrodes. In the model, the position of the metal spheres is discretized and the inhomogeneous distribution of the electric media is approximated by a resistor network. These simplifications allow us to treat a large number of particles, which is absolutely required to describe the structural changes of the system. By our simulations, behaviors on both macroscopic and microscopic levels were studied in detail.

On the macroscopic level, the behavior of the system is characterized by the conductivity, which is monitored as a function of the imposed voltage. Simulations revealed a transition from the insulating to the conducting state as the voltage increased. As a very interesting outcome of the work, simulations showed that the critical voltage of the transition exhibits a power-law behavior as a function of the system size, however, the value of the exponent is significantly different from the trivial scaling exponent of bulk systems. The nontrivial scaling of the macroscopic characteristics is the fingerprint of the complex evolution of the microstructure of the particle system with increasing voltage. Another interesting outcome of the work is that simulations also showed a power-law scaling for the saturation conductivity, although it is far from the insulatorconductor transition range. This suggested that the effective section of the conductive backbone scales with the system size by a fractal dimension even after the structure did not change with the applied field.

On the microscopic level, the dipole-dipole interaction of the particles gives rise to a cluster-cluster aggregation process, which then leads to the formation of a spanning cluster between the electrodes. The increasing value of the conductivity is the consequence of the evolution of the clusters' structure with increasing voltage. Our analysis showed that the clusters which are disconnected from the electrodes are nearly compact, however, they are strongly elongated along the field direction. The anisotropic cluster shape proved to have a self-affine character, i.e., clusters get more elongated as they grow with a power-law dependence of the aspect ratio on the linear extension. The structural evolution exhibits a transition from a phase where all clusters are relatively small compared to the system size, to another phase where a macroscopic cluster that is connected to two electrodes forms. The transitional voltage has almost the same value, and hence scaling behavior, with that of the critical voltage determined from the functional form of the conductivity. We also found that, in spite of the highly anisotropic cluster shapes, the size distribution of clusters exhibits a power-law behavior at the transition point with an exponent which falls very close to the corresponding value of percolation in $2 \mathrm{D}$.

The critical voltage can also be directly observed in experiment. The results show that both the experimental data and theoretical data can be plotted in a single straight line in the double logarithmic plot as a function of system size by fitting $\alpha / f_{0}$ appropriately. This implies that the experimental results also support the exponent $\gamma=0.23$ in Fig. 5, although the experimental data are more scattered because of the smaller system sizes [22].

\section{ACKNOWLEDGMENTS}

This work is supported by Key Item of Knowledge Innovation Project of Chinese Academy of Sciences (Grant No. KJCX2-YW-M07) and National Natural Science Foundation of China under Grants No. 10674157 and No. 10875166. This work was partly supported by the Chinese-Hungarian Intergovernmental project $\mathrm{CH}$ 27/2007. F.K. was supported by TAMOP 4.2.1-08/1-2008-003 project and by the Bolyai Janos project of HAS.
[1] Phase Transitions and Critical Phenomena, Vols. 1-20, edited by C. Domb, M. S. Green, and J. L. Lebowitz (Academic, New York, 1972-2001).

[2] M. E. Fisher, Rev. Mod. Phys. 46, 597 (1974).

[3] J. M. Yeomans, Statistical Mechanics of Phase Transitions (Oxford University Press, Oxford, UK, 1992).
[4] L. Onsager, Phys. Rev. 65, 117 (1944).

[5] C. N. Yang, Phys. Rev. 85, 808 (1952).

[6] B. M. McCoy and T. T. Wu, The Two-Dimensional Ising Model (Harvard University Press, Boston, MA, 1973).

[7] J. P. Clerc, G. Giraud, J. M. Laugier, and J. M. Luck, Adv. Phys. 39, 191 (1990). 
[8] D. J. Bergman and D. Stroud, Solid State Phys. 46, 147 (1992).

[9] D. Stauffer and A. Aharony, Introduction to Percolation Theory (Taylor \& Francis, London, 1992).

[10] I. Webman, J. Jortner, and M. H. Cohen, Phys. Rev. B 11, 2885 (1975).

[11] J. P. Straley, J. Phys. C 9, 783 (1976).

[12] A. L. Efros and B. I. Shklovskii, Phys. Status Solidi B 76, 475 (1976).

[13] D. J. Bergman and Y. Imry, Phys. Rev. Lett. 39, 1222 (1977).

[14] D. C. Hong, H. E. Stanley, A. Coniglio, and A. Bunde, Phys. Rev. B 33, 4564 (1986).

[15] W. M. Winslow, J. Appl. Phys. 20, 1137 (1949).

[16] F. E. Filisko and D. R. Gamota, Recent Advances in NonNewtonian Flows (American Society of Mechanical Engineers, New York, 1992), Vol. 153, p. 75.

[17] H. Ma, W. Wen, W. Y. Tam, and P. Sheng, Adv. Phys. 52, 343 (2003).

[18] T. C. Halsey, Science 258, 761 (1992).

[19] J. E. Stangroom, J. Stat. Phys. 64, 1059 (1991); Phys. Technol. 14, 290 (1983).

[20] R. Stanway, J. L. Sproston, and A. K. El-Wahed, Smart Mater. Struct. 5, 464 (1996).

[21] G. Georgiades and S. O. Oyadiji, J. Intell. Mater. Syst. Struct. 14, 105 (2003).

[22] W. Wen and K. Lu, Phys. Rev. E 55, R2100 (1997).

[23] H. J. Limbach, A. Arnold, B. A. Mann, and C. Holm, Comput. Phys. Commun. 174, 704 (2006).
[24] E. Lindahl, B. Hess, and D. van der Spoel, J. Mol. Model 7, 306 (2001).

[25] L. Kale, R. Skeel, M. Bhandarkar, R. Brunner, A. Gursoy, N. Krawetz, J. Phillips, A. Shinozaki, K. Varadarajan, and K. Schulten, J. Comput. Phys. 151, 283 (1999).

[26] R. W. Hockney and J. W. Eastwood, Computer Simulation Using Particles (McGraw-Hill, New York, 1981).

[27] V. Ballenegger, J. J. Cerda, O. Lenz, and C. Holm, J. Chem. Phys. 128, 034109 (2008).

[28] G. G. Batrouni, A. Hansen, and M. Nelkin, Phys. Rev. Lett. 57, 1336 (1986).

[29] F. Kun and K. F. Pal, Phys. Rev. E 57, 3216 (1998).

[30] W. Wen, X. Huang, S. Yang, K. Lu, and P. Sheng, Nat. Mater. 2, 727 (2003).

[31] W. Y. Tam, G. H. Yi, W. Wen, H. Ma, M. M. T. Loy, and P. Sheng, Phys. Rev. Lett. 78, 2987 (1997).

[32] R. Tao and J. M. Sun, Phys. Rev. Lett. 67, 398 (1991); R. Tao and Q. Jiang, ibid. 73, 205 (1994).

[33] X. Feng, Y. Deng, and H. W. J. Blote, Phys. Rev. E 78, 031136 (2008).

[34] M. E. J. Newman and R. M. Ziff, Phys. Rev. Lett. 85, 4104 (2000).

[35] M. J. Lee, Phys. Rev. E 76, 027702 (2007); 78, 031131 (2008).

[36] J. Zhang, X. Gong, C. Liu, W. Wen, and P. Sheng, Phys. Rev. Lett. 101, 194503 (2008).

[37] K. von Pfeil, M. D. Graham, D. J. Klingenberg, and J. F. Morris, Phys. Rev. Lett. 88, 188301 (2002).

[38] W. Wen, X. Huang, and P. Sheng, Soft Matter 4, 200 (2008). 